\title{
42. PHYSICAL PROPERTIES, COMPRESSIONAL-WAVE VELOCITY, AND CONSOLIDATION CHARACTERISTICS OF SLOPE SEDIMENTS, TOWNSVILLE TROUGH, NORTHEAST AUSTRALIA ${ }^{1}$
}

\author{
Min-Pen Chen, ${ }^{2}$ Jeng-Shyan Juang, ${ }^{2}$ and John Ladd ${ }^{3}$
}

\begin{abstract}
Sites 815 and 817 were drilled near the Townsville Trough during Leg 133 of the Ocean Drilling Program. The physical properties, compressional-wave velocity, and consolidation characteristics indicate that the periplatform carbonate sediments maintain more water content and lower compressional velocity near the Queensland Plateau than the clayey hemipelagic sediments, which have a clay content of up to $60 \%$. Bulk density, void ratio or porosity, water content, and compressional-wave velocity are shown to have a linear relationship with burial depth. Between 3.5 and $5 \mathrm{Ma}$ (about $100-500 \mathrm{mbsf}$ ), these physical properties maintained a constant rate vs. the depth in core because of the fast sedimentation-rate effect at Site 815. However, compressionalwave velocity still increases downward in this section. The clay content in this section causes an increase of bulk modulus and compaction effect. At Site 817 , scarce terrigenous mud content and abundant carbonate content $(88 \%-97 \%)$ cause a straight line relationship between physical properties and burial depth. During the consolidation test, we show that dominant micritic particles may cause faster acoustic velocity than sediments composed mainly of coccoliths. The bulk modulus ratio increasing rate in the clay-rich carbonate sediments is almost 4.5 times higher than in the clay-free periplatform carbonate sediments.
\end{abstract}

\section{INTRODUCTION}

Physical properties, compressional-wave velocity, and consolidation characteristics play important roles in understanding compaction history. During Leg 133, extensive physical properties and compressional-wave velocity measurements were routinely performed in the shipboard laboratory. The measured compressional-wave velocity was not in an indigenous state because the drilled sediments had already been released from the original consolidation state. Shorebased laboratory analyses attempted to measure velocity while the sediment was completely consolidated under certain loadings. Here, we review the physical-properties data and attempt to relate these properties to different environments.

A total of five sites were drilled along a north-south transact of the Townsville Trough (Fig. 1), a major physiographic east-west feature $560 \mathrm{~km}$ long that separates the Queensland and Marion Plateaus. Only Sites 815 and 817 were chosen for this study. Site 815 is located along the northern margin of the Marion Plateau, approximately $250 \mathrm{~km}$ east of the Australian mainland (Fig. 1). At the present time, reef growth is restricted to Marion Reef on the northeastern corner and Saumarez Reef at the southeastern extremity of the plateau (Davies, 1989). The upper surface of the Marion Plateau is swept by moderately strong currents such that only hemipelagic sediments have accumulated (Davies, McKenzie, Palmer-Julson, et al., 1991). Site 815 is located in approximately $465 \mathrm{~m}$ of water on the southern edge of the Townsville Trough. Hole $815 \mathrm{~A}$ was drilled to a depth of 473.5 meters below seafloor (mbsf). The advanced piston corer (APC) was replaced by the extended core-barrel corer (XCB) at about $226 \mathrm{mbsf}$. Site 817 is situated (Fig. 1) on the northern side of the Townsville Trough, southwest of the Tregrosse/Lihou/Coringa bank complex. Site 817 was drilled in about $1016 \mathrm{~m}$ of water to a depth of $700 \mathrm{~m}$.

\footnotetext{
'McKenzie, J.A., Davies, P.J., Palmer-Julson, A., et al.. 1993. Proc. ODP, Sci. Results, 133: College Station, TX (Ocean Drilling Program).

${ }^{2}$ Institute of Oceanography, National Taiwan University, Taipei, Taiwan, Republic of China.

${ }^{3}$ Lamont-Doherty Earth Observatory, Columbia University, Palisades, NY 10964,
}

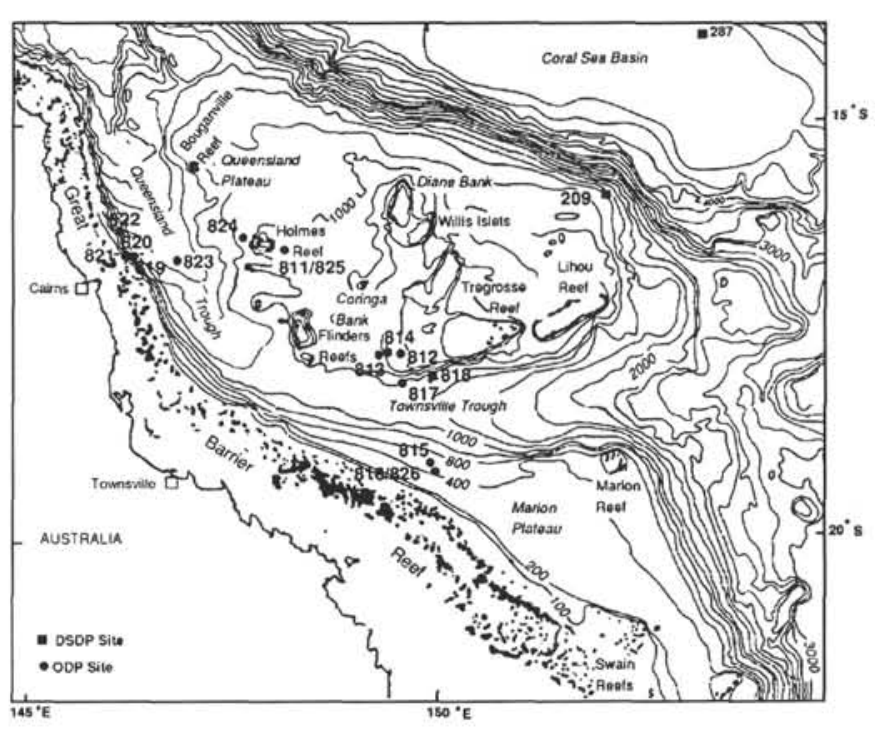

Figure 1. Position of Sites 815 and 817 and other Leg 133 sites off northeastern Australia (from Davies, McKenzie, Palmer-Julson, et al., 1991).

Using double APC-drilling, complete sections were recovered to a depth of 204 mbsf.

At Site 815 , the following lithologic units were defined:

1. Unit I (0-73.3 mbsf): Foraminifer nannofossil ooze with little or no clay (Pleistocene to late early Pliocene).

2. Unit II (73.3-280.0 mbsf): Nannofossil ooze with clay to clayey nannofossil mixed sediment, documenting considerable variation in clay content of up to $60 \%$ (early Pliocene).

3. Unit III (280.5-348.4 mbsf): Nannofossil chalk with evidence of slumping (early Pliocene).

4. Unit IV (348.4-425.3 mbsf): Foraminifer nannofossil chalk to nannofossil foraminifer chalk, showing preservation of densely distributed burrows (early early Pliocene to late late Miocene). 
5. Unit V (425.3-444.5 mbsf): Dolomitized foraminifer packstone to rudstone (late Miocene).

6. Unit VI (454.2-463.8 mbsf): Dolomitized benthic foraminifer rudstone to floatstone within a foraminifer packstone (latest early to early middle Miocene).

At Site 817, the following lithologic units were defined:

1. Unit I (0-200.8 mbsf): Periplatform over nannofossil ooze with minor calciturbidites and slumps (Pleistocene to late Miocene).

2. Unit II (200.8-426.7 mbsf): Nannofossil ooze with decreasing bioclastic supply (late Miocene to middle Miocene).

3. Unit III (426.7-666.8 mbsf): Redeposited bioclastic sands and nannofossil ooze (middle Miocene to latest early Miocene or older).

\section{METHODS}

Sediment physical properties measurements were routinely performed aboard ship on all Leg 133 cores according to the general procedures outlined by Boyce (1976). The parameters measured were index properties (wet-bulk density, grain density, water content, porosity), vane shear strength, compressional-wave velocity, thermal conductivity, and formation factor. Shore-based laboratory analyses consisted of a total of 10 vertical consolidation and compressionalwave velocity measurements on all whole-round samples: 133-815B$1 \mathrm{H}-4,140-150 \mathrm{~cm}$ (5.9-6.0 mbsf); 133-815B-2H-4, 140-150 cm (13.8-13.9 mbsf); 133-815B-3H-4, 140-150 cm (23.3-23.4 mbsf); 133-815B-4H-4, $140-150 \mathrm{~cm}$ (32.8-32.9 mbsf); 133-817B-3H-4, $140-150 \mathrm{~cm}$ (19.9-20.0 mbsf); 133-817B-6H-4, 140-150 cm (48.4 48.5 mbsf); $133-817 \mathrm{~B}-10 \mathrm{H}-4,140-150 \mathrm{~cm}$ (86.4-86.5 mbsf); $133-$ 817B-13H-4, 140-150 cm (114.9-115.0 mbsf); 133-817B-16H-4, $140-150 \mathrm{~cm}$ (143.4-143.5 mbsf); and 133-817B-20H-4, 140-150 cm (181.4-181.5 mbsf). Each sample was also used to determine the grain size, water content, wet-bulk density, and initial void ratio prior to all consolidation testing. These samples were sealed tightly in the refrigerator, and their degree of saturation should have been preserved in $100 \%$ condition; therefore, the tests did not employ a back-pressure system to achieve saturation. A soil test consolidation frame with a fixed ring consolidometer $6.35 \mathrm{~cm}$ in diameter and $2.54 \mathrm{~cm}$ high was used. Loads were added incrementally in $24-\mathrm{hr}$ periods. The initial vertical pressure was $0.01 \mathrm{~kg} / \mathrm{cm}^{2}$. Each subsequent load was doubled until a total vertical pressure of $25.6 \mathrm{~kg} / \mathrm{cm}^{2}$ was reached. The sample was then unloaded at a ratio of $1: 4$. The effective overburden pressure, $P^{\prime}$, acting on the sample in place, is the combined grain density of the overlying sediment minus the density of seawater (McClelland,1967). The $P^{\prime}$ then can be calculated from:

$$
P^{\prime}=\sum r_{w}\left[\left(G-r_{w}\right) /(1+e)\right] d z,
$$

where $G$ equals the grain density of sediment, $e$ is the void ratio, $r_{w}$ is the density of seawater ( 1.025 as an assumed average), and $d z$ is the interval of depth.

Grain sizes were determined for each of the studied whole-round samples by separating the sand-size fraction with wet-sieving and the silt and clay size fraction using pipette analysis (Folk, 1974). Sodium hexametaphosphate was used for the dispersed medium.

Determination of carbonate content in the samples was made according to the weight-loss method outlined by Molnia (1974); however, before that, the carbonate material was dissolved by $1 \mathrm{~N}$ hydrochloric acid and the salt in each sample was washed out with distilled water.

Piezoelectric transducers were mounted in the cap and base of the consolidation container. Both transducers were tightly contacted with porous stones completely saturated with filtered seawater. A frequency of $500 \mathrm{kHz}$ was used to maintain the same frequency as was used in the shipboard laboratory. The amplified signals were fed to an oscilloscope along with synchronizing signals from the pulse generator to ensure a stable waveform on the screen for visual observations. The transit times were measured with the help of a movable marker and are accurate to $0.01 \mu \mathrm{s}$.

\section{RESULTS}

Carbonate content and grain-size distribution data are presented in Tables 1 and 2. Carbonate contents at Site 815 vary from $92 \%$ at 5.5 mbsf to a minimum value of $33 \%$ at 131 mbsf. At Site 817 , carbonate contents vary between $97 \%$ and $88 \%$ (Fig. 2). The mean grain sizes in the four sections of Hole $815 \mathrm{~B}$ are coarser than the sections of Hole $817 \mathrm{~B}$. At Site 815 , the sand content varies from $37 \%$ in Section 133-815B-4H-4 to 50\% in Section 133-815B-1H-4; however, the carbonate content is $70.3 \%$ in the former section and $88.5 \%$ in the latter section. These carbonate contents are less than the contents in Hole $817 \mathrm{~B}$, where the sand content is generally less than $10 \%$, except for Section 133-817B-20H-4, with $14 \%$. The clay content maintains more than $60 \%$ in the six sections of Hole $817 \mathrm{~B}$. The results of grain-size distribution and carbonate content analyses indicate that calcareous micrite is much more abundant in Hole $817 \mathrm{~B}$ and calcareous microfossils, such as foraminifers, are more abundant in Hole $815 \mathrm{~B}$. One can reasonably say that within the top 110 -m section, the physical-properties in Hole $815 \mathrm{~B}$ change faster than those in Hole 817B (Figs. 2, 3, and 4).

The bulk density at Site 817 increases steadily from a minimum value of $1.57 \mathrm{~g} / \mathrm{cm}^{3}$ just beneath the seafloor to a maximum value of $2 \mathrm{~g} / \mathrm{cm}^{3}$ at $205 \mathrm{mbsf}$. However, the bulk density at Site 815 increases rapidly from 1.64 to $1.8 \mathrm{~g} / \mathrm{cm}^{3}$ within the top $100-\mathrm{m}$ layer, and then, it maintains a high variation between 1.8 and $2.1 \mathrm{~g} / \mathrm{cm}^{3}$ between 110 and 330 mbsf (Fig. 2). At both sites, variations in water content and porosity show a reversed pattern with bulk density (Figs. 2 and 3). At Site 815 , these sharply decreasing rates occurred only within the period of low sedimentation rate, 1.7 to $3.2 \mathrm{~cm} / \mathrm{k}$.y. (Gartner, this volume). However, between 110 and $330 \mathrm{mbsf}$, the sedimentation rate increases up to $38.5 \mathrm{~cm} / \mathrm{k} . \mathrm{y}$. (Gartner, this volume); its porosity maintains a more or less constant rate in this period (Fig. 3).

Compressional-wave velocities of the sediments at both Sites 815 and 817 indicate that the greater the terrigenous mud content, the faster the velocity (Fig. 4). Although the curves of compressional-

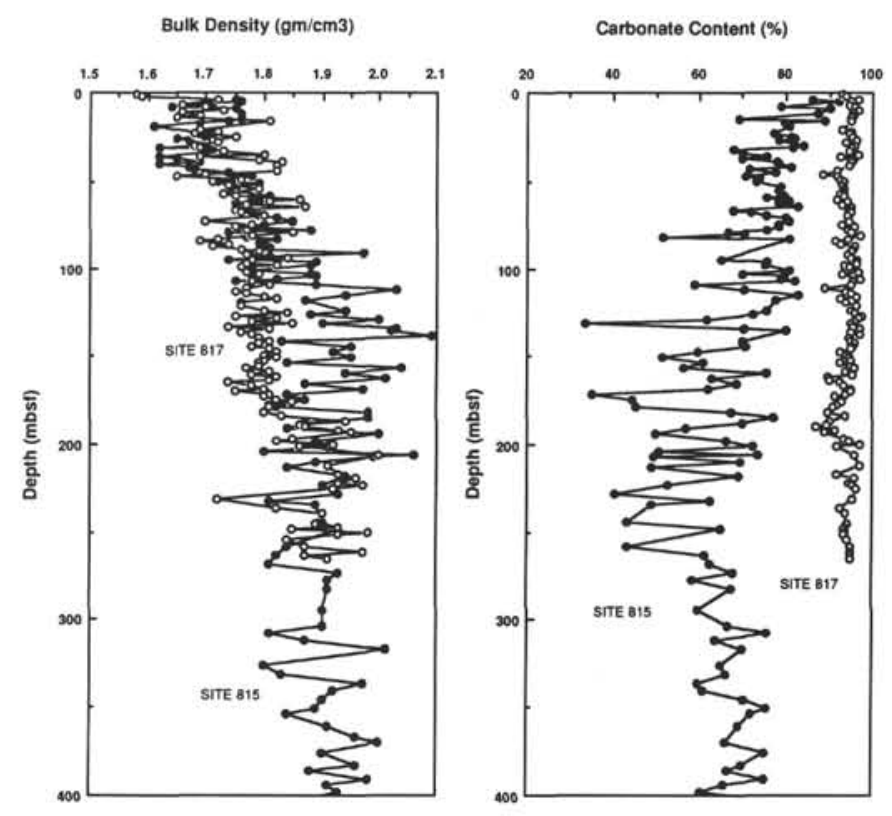

Figure 2. Sediment carbonate content and bulk density vs. depth at Sites 815 and 817 . 
Table 1. Lithologic characteristics of samples from Hole 815B and 817B.

\begin{tabular}{|c|c|c|c|c|}
\hline Section & $\begin{array}{l}\text { Depth } \\
\text { (mbsf) }\end{array}$ & Age & Constituents & $\begin{array}{l}\text { Carbonate } \\
\text { content }(\%)\end{array}$ \\
\hline $\begin{array}{l}133-815 \mathrm{~A}-1 \mathrm{H}-1 \\
133-815 \mathrm{~B}-1 \mathrm{H}-4\end{array}$ & $\begin{array}{l}1.35 \\
5.93\end{array}$ & late Pleistocene & $\begin{array}{l}60 \% \text { foraminifers; } \\
16 \% \text { nannofossils; } 10 \% \text { bioclasts } \\
5 \% \text { pteropods; } 3 \% \text { micrite; } \\
3 \% \text { quartz; } 3 \% \text { spicules }\end{array}$ & $a_{88.2}^{91}$ \\
\hline $\begin{array}{l}133-815 \mathrm{~A}-2 \mathrm{H}-4 \\
133-815 \mathrm{~B}-2 \mathrm{H}-4\end{array}$ & $\begin{array}{l}11.1 \\
13.83\end{array}$ & early Pleistocene & $\begin{array}{l}40 \% \text { foraminifers; } \\
30 \% \text { nannofossils; } 20 \% \text { oxide; } \\
7 \% \text { calcite; } 1 \% \text { quartz; } 1 \% \text { spicules }\end{array}$ & $\begin{array}{r}87.2 \\
a_{86.9}\end{array}$ \\
\hline $\begin{array}{l}133-815 \mathrm{~A}-3 \mathrm{H}-1 \\
133-815 B-3 \mathrm{H}-4\end{array}$ & $\begin{array}{l}17.41 \\
23.33\end{array}$ & $\begin{array}{l}\text { early early } \\
\text { Pleistocene }\end{array}$ & $\begin{array}{l}15 \% \text { foraminifers; } \\
52 \% \text { nannofossils; } 20 \% \text { bioclasts; } \\
5 \% \text { micrite; } 3 \% \text { quartz }\end{array}$ & $\begin{array}{l}79.5 \\
a_{85.3}\end{array}$ \\
\hline $\begin{array}{l}133-815 \mathrm{~A}-4 \mathrm{H}-6 \\
133-815 B-4 \mathrm{H}-4\end{array}$ & $\begin{array}{l}32.35 \\
32.83\end{array}$ & late Pliocene & $\begin{array}{l}15 \% \text { foraminifers; } \\
55 \% \text { nannofossils; } 20 \% \text { bioclasts; } \\
5 \% \text { quartz; } 3 \% \text { spicules }\end{array}$ & $\begin{array}{l}69.9 \\
a_{70.3}\end{array}$ \\
\hline $\begin{array}{l}133-817 \mathrm{~A}-3 \mathrm{H}-3 \\
133-817 \mathrm{~B}-3 \mathrm{H}-4\end{array}$ & $\begin{array}{l}19.12 \\
19.93\end{array}$ & late Pleistocene & $\begin{array}{l}10 \% \text { foraminifers; } \\
20 \% \text { nannofossils; } 15 \% \text { bioclasts; } \\
45 \% \text { micrite; } 5 \% \text { calcite; } \\
3 \% \text { spicules; } 2 \% \text { lithoclast }\end{array}$ & $\begin{array}{l}93.2 \\
a_{94.4}\end{array}$ \\
\hline $\begin{array}{l}133-817 \mathrm{~A}-6 \mathrm{H}-4 \\
133-817 \mathrm{~B}-6 \mathrm{H}-4\end{array}$ & $\begin{array}{l}48.28 \\
48.43\end{array}$ & early Pleistocene & $\begin{array}{l}10 \% \text { foraminifers; } \\
20 \% \text { nannofossils; } 15 \% \text { bioclasts; } \\
47 \% \text { micrite; } 5 \% \text { aragonite; } \\
3 \% \text { quartz }\end{array}$ & $\begin{array}{l}92.6 \\
a_{96.4}\end{array}$ \\
\hline $\begin{array}{l}133-817 \mathrm{~A}-10 \mathrm{H}-4 \\
133-817 \mathrm{~B}-10 \mathrm{H}-4\end{array}$ & $\begin{array}{l}86.44 \\
86.43\end{array}$ & late Pliocene & $\begin{array}{l}3 \% \text { foraminifers; } \\
35 \% \text { nannofossils; } 2 \% \text { bioclasts; } \\
50 \% \text { micrite; } 5 \% \text { spicules; } \\
3 \% \text { lithoclast; } 2 \% \text { quartz }\end{array}$ & $\begin{array}{l}95.8 \\
a_{98.1}\end{array}$ \\
\hline $\begin{array}{l}133-817 \mathrm{~A}-13 \mathrm{H}-5 \\
133-817 \mathrm{~B}-13 \mathrm{H}-4\end{array}$ & $\begin{array}{l}115.3 \\
114.93\end{array}$ & early Pliocene & $\begin{array}{l}15 \% \text { foraminifers; } \\
20 \% \text { nannofossils; } 13 \% \text { bioclasts; } \\
35 \% \text { micrite; } 8 \% \text { inorganic calcite; } \\
9 \% \text { spicules }\end{array}$ & $\begin{array}{l}92.8 \\
a_{97.9}\end{array}$ \\
\hline $\begin{array}{l}133-817 \mathrm{~A}-16 \mathrm{H}-2 \\
133-817 \mathrm{~B}-16 \mathrm{H}-4\end{array}$ & $\begin{array}{l}141.5 \\
143.43\end{array}$ & early Pliocene & $\begin{array}{l}17 \% \text { foraminifers; } \\
37 \% \text { nannofossils; } 15 \% \text { bioclasts; } \\
20 \% \text { micrite; } 6 \% \text { inorganic calcite; } \\
5 \% \text { spicules; }\end{array}$ & $\begin{array}{l}95.2 \\
a_{97.9}\end{array}$ \\
\hline $\begin{array}{l}133-817 \mathrm{~A}-2 \mathrm{OH}-3 \\
133-817 \mathrm{~B}-20 \mathrm{H}-4\end{array}$ & $\begin{array}{l}180.5 \\
181.43\end{array}$ & late Miocene & $\begin{array}{l}25 \% \text { foraminifers; } \\
54 \% \text { nannofossils; } 7 \% \text { bioclasts; } \\
3 \% \text { micrite; } 5 \% \text { inorganic calcite; } \\
6 \% \text { spicules }\end{array}$ & $\begin{array}{l}92.5 \\
a_{94.3}\end{array}$ \\
\hline
\end{tabular}

a

Measured in the shore-based laboratory 
Table 2. Results of grain-size analyses of samples from Holes 815B and 817B.

\begin{tabular}{lrcccccc}
\hline Section & $\begin{array}{r}\text { Sand } \\
(\%)\end{array}$ & $\begin{array}{c}\text { Silt } \\
(\%)\end{array}$ & $\begin{array}{c}\text { Clay } \\
(\%)\end{array}$ & $\begin{array}{c}\text { Mean size } \\
(\phi)\end{array}$ & $\begin{array}{c}\text { Std. dev. } \\
(\phi)\end{array}$ & Skewness & Kurtosis \\
\hline $133-$ & & & & & & & \\
$-815 \mathrm{~B}-1 \mathrm{H}-4$ & 50.00 & 20.54 & 29.46 & 5.61 & 3.48 & 0.57 & 0.76 \\
$-815 \mathrm{~B}-2 \mathrm{H}-4$ & 43.65 & 17.38 & 38.97 & 6.09 & 3.68 & 0.25 & 0.64 \\
$-815 \mathrm{~B}-3 \mathrm{H}-4$ & 44.44 & 17.36 & 38.21 & 6.20 & 3.81 & 0.30 & 0.63 \\
$-815 \mathrm{~B}-4 \mathrm{H}-4$ & 37.45 & 17.33 & 45.22 & 6.97 & 4.29 & -0.03 & 0.62 \\
$-817 \mathrm{~B}-3 \mathrm{H}-4$ & 48.41 & 20.03 & 31.56 & 6.19 & 2.95 & 0.79 & 0.62 \\
$-817 \mathrm{~B}-6 \mathrm{H}-4$ & 10.75 & 27.46 & 61.79 & 8.34 & 2.81 & -0.35 & 1.05 \\
$-817 \mathrm{~B}-10 \mathrm{H}-4$ & 9.01 & 27.68 & 63.30 & 8.24 & 2.46 & -0.35 & 1.09 \\
$-817 \mathrm{~B}-13 \mathrm{H}-4$ & 9.34 & 27.35 & 63.30 & 8.26 & 2.49 & -0.29 & 1.24 \\
$-817 \mathrm{~B}-16 \mathrm{H}-4$ & 8.38 & 21.78 & 69.84 & 8.58 & 2.56 & -0.34 & 1.20 \\
$-817 \mathrm{~B}-20 \mathrm{H}-4$ & 14.46 & 25.29 & 60.25 & 7.85 & 2.96 & -0.36 & 1.01 \\
\hline
\end{tabular}
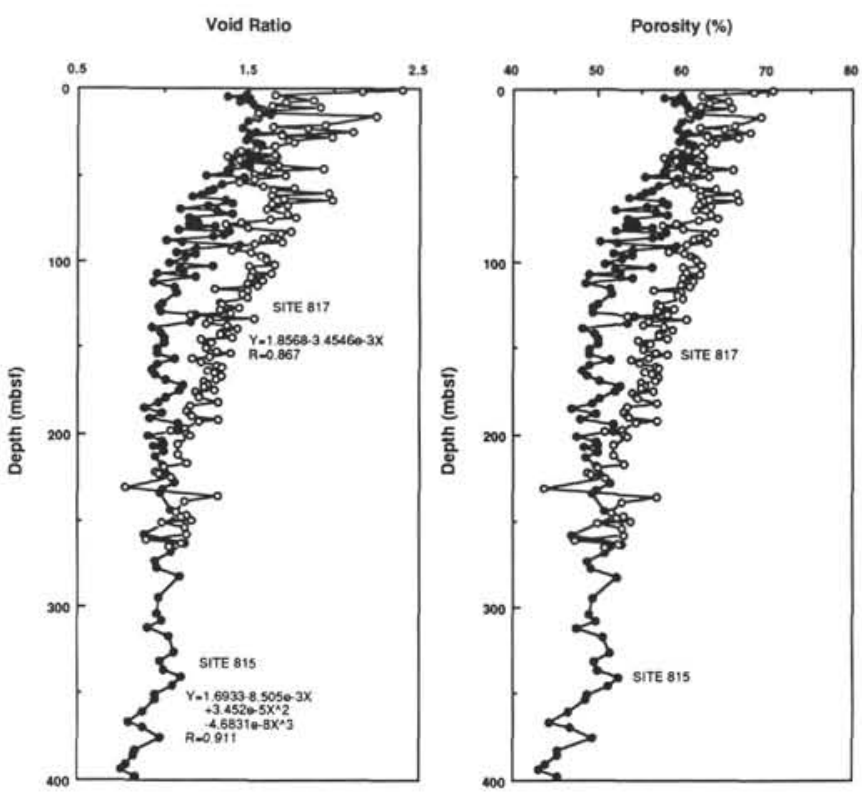

Figure 3. Void ratio and porosity vs. depth of sediments at Sites 815 and 817.

wave velocity vs. stress at the two sites are offset about $100 \mathrm{~m} / \mathrm{s}$ (Fig. 5) during the low-stress loading period within the shallow burial depth, they emerge together at highly applied normal stress eventually, for instance, above $40 \mathrm{~kg} / \mathrm{cm}^{2}$ (Fig. 5). At Site 815 , the compressional-wave velocity increasing rate has not been interrupted by the almost constant porosity between 110 and $330 \mathrm{mbsf}$, because sonic velocity is not only dependent on bulk density, but is also strongly affected by bulk modulus (incompressibility). Bulk modulus $(K)$ is a function of compressional-wave velocity $(V p)$, shear-wave velocity $(V s)$, and bulk density $(\rho), K=\rho\left(V p^{2}-3 / 4 V s^{2}\right)$. In this study, shear-wave velocity has been estimated using Hamilton's empirical equations (1979). Bulk modulus is a main parameter for establishing the original state. Consolidation results (Figs. 6 and 7) show that the diminution of void ratio during the compaction test at $32.8 \mathrm{mbsf}$ of Site 815 is still greater than the sediments from 19.9 mbsf of Site 817 . The carbonate content of the former sediments is $70.3 \%$, and the latter is $94.4 \%$. The former grain-size (mean $=6.97 \phi)$ is a little finer than the latter $($ mean $=6.19 \phi)($ Table 2$)$.

\section{DISCUSSION}

Comparing the void ratios of four consolidated sediments at Site 815 , the smallest value appears in the shallowest depth, 1.31 at 5.93

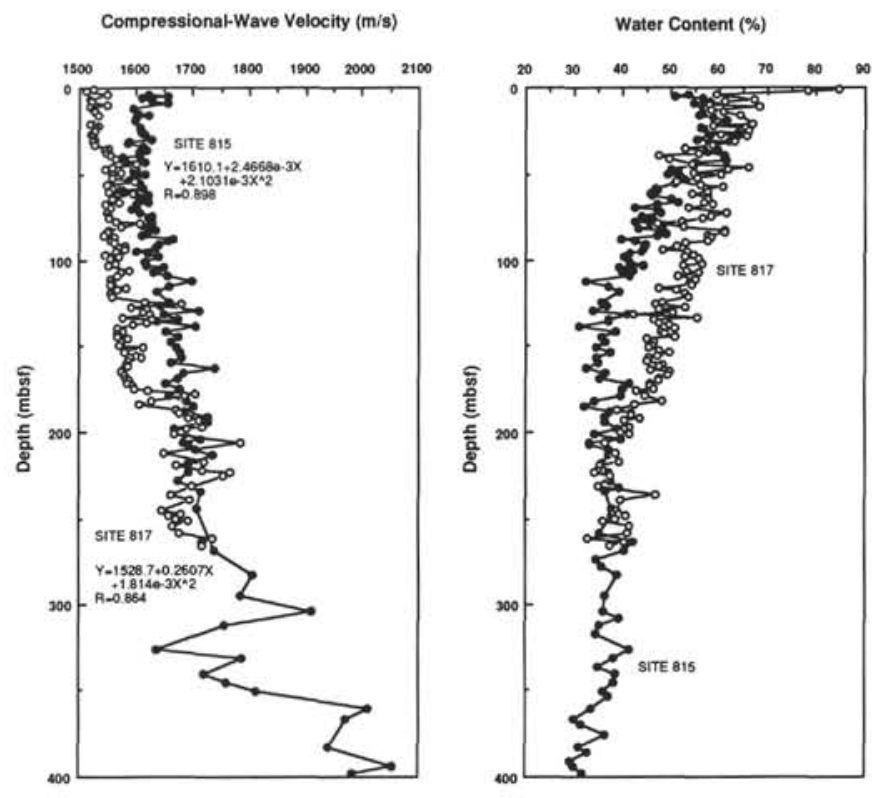

Figure 4. Compressional-wave velocity and water content of sediments vs. depth at Sites 815 and 817.

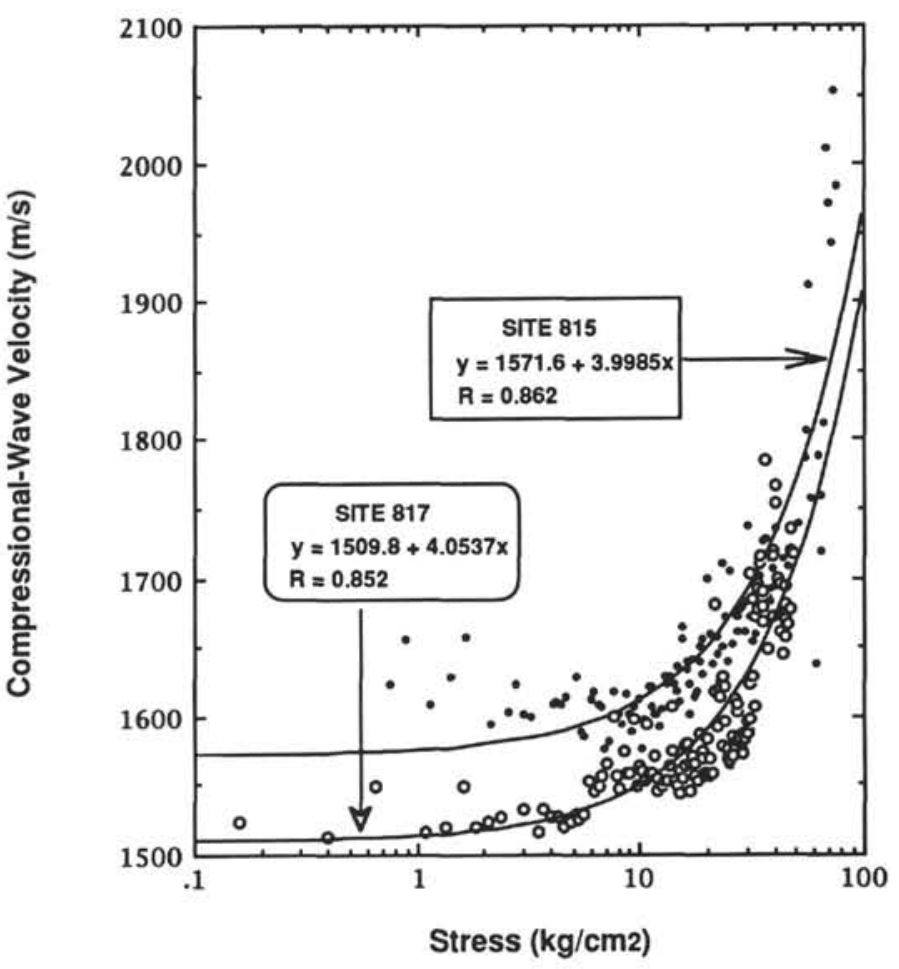

Figure 5. Compressional-wave velocity vs. overburden pressure at Sites 815 and 817 .

mbsf. This abnormal phenomenon also was indicated in the results of shipboard measurements (Figs. 3 and 6). This decreasing feature possibly results from the collapsing effect created when the highporosity sediment was drilled and loaded onto the ship. Below 13.83 mbsf $\left(0.94 \mathrm{~kg} / \mathrm{cm}^{2}\right)$, this collapsing effect does not occur. This sediment shows a higher void ratio, 1.58, and faster compressional-wave velocity, $1615 \mathrm{~m} / \mathrm{s}$, than the shallower sediment (Table 3).

At Site 817, its sedimentation rate was maintained at 1.6 to 6.2 $\mathrm{cm} / \mathrm{k}$.y. (Gartner, this volume). As a matter of fact, the void ratio from 


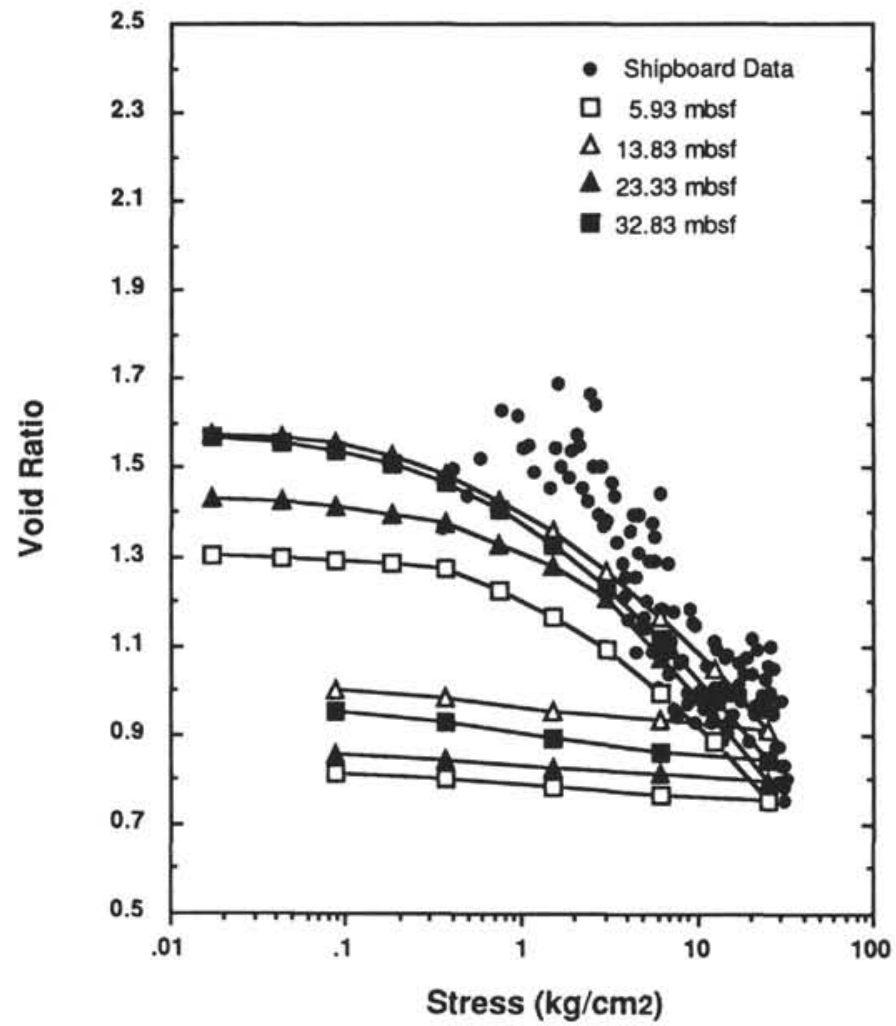

Figure 6. Void ratio vs. effective stress for Site 815 consolidation samples and comparison of shipboard measurements.

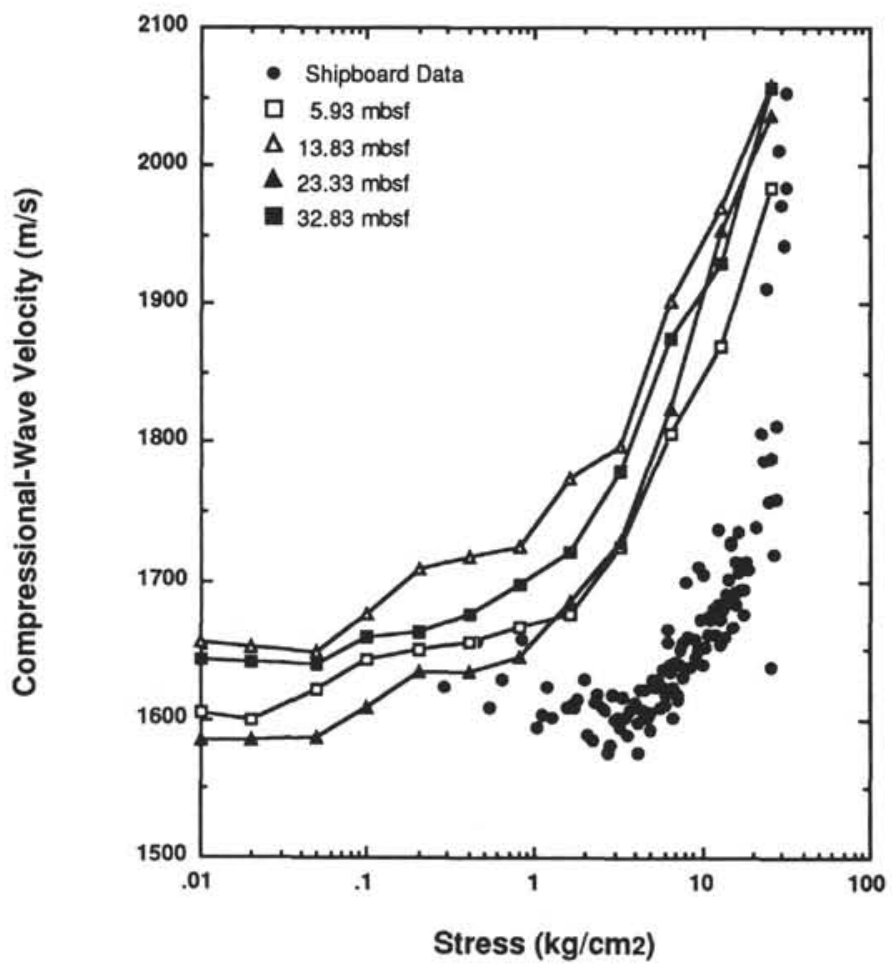

Figure 7. Compressional-wave velocity vs. effective stress for Site 815 consolidation samples and comparison of shipboard measurements. The $P^{\prime}$ then can be calculated from: $P^{\prime}=\sum r_{w}\left[\left(G-r_{w}\right) /(1+e)\right] d z$, where $G$ equals the grain density of sediment, $e$ is the void ratio, $r_{w}$ is the density of seawater (1.025 as an assumed average), and $d z$ is the interval of depth.

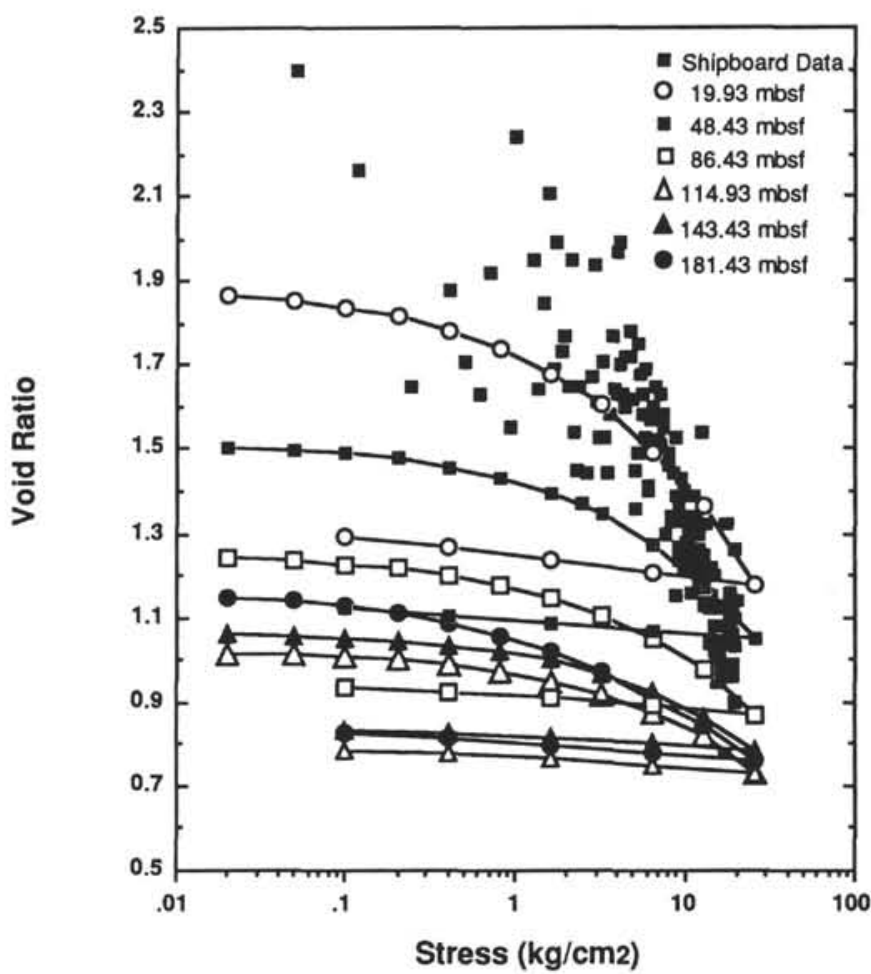

Figure 8. Void ratio vs. effective stress for Site 817 consolidation samples and comparison of shipboard measurements.

Site 817 has a clear and straight relationship with burial depth, $Y$ (void ratio $)=1.8568-3.4546 \times 10^{-3} X($ depth $),(r=0.867)$. By contrast, at Site 815 , these relationships become more complicated: $Y$ (void ratio) $=1.6933-8.504 \times 10^{-3} X$ (depth) $+3.452 \times 10^{-5} X^{2}-4.631 \times 10^{-8} X^{3}$, $(r=0.911$ ) (Fig. 3). At Site 817, the implication is that the high carbonate content of the periplatform sediments was heavily bioturbated by the benthic organisms during periods of slow depositional rates. At Site 815, during the fast depositional rate period, pore waters did not have enough time to be dispersed beneath the overlain fine-grained terrigenous mud.

The shapes of the e-log $p$ curves are typical of curves for calcareous sediments found by other scientists (Bryant et al., 1974; Demars, 1982; Lavoie, 1988; Lee, 1982). Trends of these curves are the same as the empirical equations at both sites (Figs. 6 and 7). The measured compressional-wave velocities in each loading also show the same pattern (Figs. 8 and 9). The offsets of measuring curves from the consolidation test to the empirical equations of the shipboard measuring data result from compaction effects.

The carbonate contents for Site 817 consolidation samples are higher than $90 \%$. Before consolidation testing, the initial compressional-wave velocity (Fig. 7) was divided into three levels: below $1550 \mathrm{~m} / \mathrm{s}$, closing $1600 \mathrm{~m} / \mathrm{s}$, and closing $1650 \mathrm{~m} / \mathrm{s}$. The shallow sediment at $48.83 \mathrm{mbsf}$ has a faster velocity, $1594 \mathrm{~m} / \mathrm{s}$. In contrast, the deeper sediments at 86.43 and $143.43 \mathrm{mbsf}$ have slower velocities, 1542 and $1529 \mathrm{~m} / \mathrm{s}$, respectively. This is because the sediment at 48.83 mbsf is micrite ooze, but it is nannofossil ooze at 86.43 and 143.43 mbsf (Davies, McKenzie, Palmer-Julson, et al., 1991). The 2- to $8-\mu \mathrm{m}$-sized platy coccoliths are dominant in the latter sediments, which may have caused low bulk modulus. Nevertheless, the former sediment contains abundant micritic particles, which may be filled into the voids within and between carbonate microfossils and thus caused a higher bulk modulus.

We used the bulk modulus ratio (Fig. 10) to indicate the compaction difference between the shipboard and shore-based compres- 
Table 3. Consolidation test results of samples from Holes 815B and 817B.

\begin{tabular}{lcccccc}
\hline $\begin{array}{c}\text { Pressure } \\
\left(\mathrm{kg} / \mathrm{cm}^{2}\right)\end{array}$ & $\begin{array}{c}C v \\
\left(\mathrm{~cm}^{2} / \mathrm{s}\right)\end{array}$ & $\begin{array}{c}A v \\
\left(\mathrm{~cm}^{2} / \mathrm{kg}\right)\end{array}$ & $\begin{array}{c}M v \\
\left(\mathrm{~cm}^{2} / \mathrm{kg}\right)\end{array}$ & $\begin{array}{c}k \\
(\mathrm{~cm} / \mathrm{s})\end{array}$ & $e$ & $\begin{array}{c}\text { P-wave } \\
\text { velocity } \\
(\mathrm{m} / \mathrm{s})\end{array}$ \\
\hline \multicolumn{2}{l}{ Section $133-815 \mathrm{~B}-1 \mathrm{H}-4$} & & & & & \\
0 & & & & & & \\
0.01 & & & & & 1.31 & 1601 \\
0.02 & & $3.04 \mathrm{E}-01$ & $1.32 \mathrm{E}-01$ & & 1.31 & 1605 \\
0.05 & $5.61 \mathrm{E}-03$ & $1.58 \mathrm{E}-01$ & $6.87 \mathrm{E}-02$ & $1.11 \mathrm{E}-06$ & 1.30 & 1617 \\
0.1 & $5.51 \mathrm{E}-03$ & $8.52 \mathrm{E}-02$ & $3.71 \mathrm{E}-02$ & $1.65 \mathrm{E}-06$ & 1.29 & 1713 \\
0.2 & $7.39 \mathrm{E}-03$ & $8.65 \mathrm{E}-02$ & $3.77 \mathrm{E}-02$ & $8.03 \mathrm{E}-07$ & 1.29 & 1754 \\
0.4 & $1.24 \mathrm{E}-02$ & $7.19 \mathrm{E}-02$ & $3.14 \mathrm{E}-02$ & $1.12 \mathrm{E}-06$ & 1.27 & 1776 \\
0.8 & $1.20 \mathrm{E}-02$ & $1.12 \mathrm{E}-01$ & $4.95 \mathrm{E}-02$ & $1.72 \mathrm{E}-06$ & 1.23 & 1776 \\
1.6 & $1.79 \mathrm{E}-02$ & $7.72 \mathrm{E}-02$ & $3.47 \mathrm{E}-02$ & $1.79 \mathrm{E}-06$ & 1.16 & 1814 \\
3.2 & $1.69 \mathrm{E}-02$ & $4.50 \mathrm{E}-02$ & $2.08 \mathrm{E}-02$ & $1.01 \mathrm{E}-06$ & 1.09 & 1828 \\
6.4 & $2.03 \mathrm{E}-02$ & $3.03 \mathrm{E}-02$ & $1.45 \mathrm{E}-02$ & $8.46 \mathrm{E}-07$ & 1.00 & 1951 \\
12.8 & $2.49 \mathrm{E}-02$ & $1.70 \mathrm{E}-02$ & $8.52 \mathrm{E}-03$ & $6.12 \mathrm{E}-07$ & 0.89 & 2012 \\
25.6 & $3.15 \mathrm{E}-02$ & $1.06 \mathrm{E}-02$ & $5.61 \mathrm{E}-03$ & $5.12 \mathrm{E}-07$ & 0.75 & 2055
\end{tabular}

Section $133-815 \mathrm{~B}-2 \mathrm{H}-4$

$\begin{array}{lllllll}0 & & & & & 1.58 & 1615 \\ 0.01 & & & & & 1.58 & 1655 \\ 0.02 & & 1.96 \mathrm{E}-01 & 7.62 \mathrm{E}-02 & & 1.58 & 1653 \\ 0.05 & 1.52 \mathrm{E}-03 & 2.31 \mathrm{E}-01 & 8.98 \mathrm{E}-02 & 3.62 \mathrm{E}-07 & 1.57 & 1649 \\ 0.1 & 4.18 \mathrm{E}-03 & 2.70 \mathrm{E}-01 & 1.05 \mathrm{E}-01 & 1.17 \mathrm{E}-06 & 1.56 & 1675 \\ 0.2 & 9.25 \mathrm{E}-03 & 2.82 \mathrm{E}-01 & 1.10 \mathrm{E}-01 & 2.71 \mathrm{E}-06 & 1.53 & 1708 \\ 0.4 & 1.30 \mathrm{E}-02 & 2.08 \mathrm{E}-01 & 8.24 \mathrm{E}-02 & 2.84 \mathrm{E}-06 & 1.49 & 1718 \\ 0.8 & 1.54 \mathrm{E}-02 & 1.49 \mathrm{E}-01 & 5.98 \mathrm{E}-02 & 2.44 \mathrm{E}-06 & 1.43 & 1724 \\ 1.6 & 1.84 \mathrm{E}-02 & 8.64 \mathrm{E}-02 & 3.56 \mathrm{E}-02 & 1.75 \mathrm{E}-06 & 1.36 & 1774 \\ 3.2 & 2.25 \mathrm{E}-02 & 5.46 \mathrm{E}-02 & 2.32 \mathrm{E}-02 & 1.39 \mathrm{E}-06 & 1.27 & 1797 \\ 6.4 & 2.80 \mathrm{E}-02 & 3.65 \mathrm{E}-02 & 1.61 \mathrm{E}-02 & 1.20 \mathrm{E}-06 & 1.15 & 1902 \\ 12.8 & 2.53 \mathrm{E}-02 & 1.62 \mathrm{E}-02 & 7.53 \mathrm{E}-03 & 5.06 \mathrm{E}-07 & 1.05 & 1969 \\ 25.6 & 3.24 \mathrm{E}-02 & 1.06 \mathrm{E}-02 & 5.19 \mathrm{E}-03 & 4.47 \mathrm{E}-07 & 0.91 & 2058\end{array}$

Section $133-815 \mathrm{~B}-3 \mathrm{H}-4$

\begin{tabular}{|c|c|c|c|c|c|c|}
\hline \multicolumn{7}{|c|}{1.47} \\
\hline 0.01 & & & & & 1.44 & 1623 \\
\hline 0.02 & & $1.05 \mathrm{E}+00$ & $4.29 \mathrm{E}-01$ & & 1.43 & 1634 \\
\hline 0.05 & & $2.90 \mathrm{E}-01$ & $1.19 \mathrm{E}-01$ & & 1.42 & 1634 \\
\hline 0.1 & $9.00 \mathrm{E}-03$ & $2.42 \mathrm{E}-01$ & $1.00 \mathrm{E}-01$ & $2.60 \mathrm{E}-06$ & 1.41 & 1684 \\
\hline 0.2 & $5.70 \mathrm{E}-03$ & $1.39 \mathrm{E}-01$ & $5.76 \mathrm{E}-02$ & $9.46 \mathrm{E}-07$ & 1.40 & 1704 \\
\hline 0.4 & $6.45 \mathrm{E}-03$ & $1.07 \mathrm{E}-01$ & $4.45 \mathrm{E}-02$ & $8.26 \mathrm{E}-07$ & 1.37 & 1720 \\
\hline 0.8 & $1.02 \mathrm{E}-02$ & $1.16 \mathrm{E}-01$ & $4.87 \mathrm{E}-02$ & $1.43 \mathrm{E}-06$ & 1.33 & 1726 \\
\hline 1.6 & $9.74 \mathrm{E}-03$ & $6.24 \mathrm{E}-02$ & $2.68 \mathrm{E}-02$ & $7.52 \mathrm{E}-07$ & 1.28 & 1729 \\
\hline 3.2 & $1.38 \mathrm{E}-02$ & $4.39 \mathrm{E}-02$ & $1.93 \mathrm{E}-02$ & $7.66 \mathrm{E}-07$ & 1.21 & 1815 \\
\hline 6.4 & $1.59 \mathrm{E}-02$ & $4.23 \mathrm{E}-02$ & $1.91 \mathrm{E}-02$ & $8.78 \mathrm{E}-07$ & 1.07 & 1857 \\
\hline 12.8 & $1.10 \mathrm{E}-02$ & $2.10 \mathrm{E}-02$ & $1.01 \mathrm{E}-02$ & $3.21 \mathrm{E}-07$ & 0.94 & 1958 \\
\hline 25.6 & $1.38 \mathrm{E}-02$ & $1.09 \mathrm{E}-02$ & $5.65 \mathrm{E}-03$ & $2.24 \mathrm{E}-07$ & 0.80 & 2052 \\
\hline \multicolumn{7}{|c|}{ Section $133-815 B-4 H-4$} \\
\hline 0 & & & & & 1.58 & 1651 \\
\hline 0.01 & & & & & 1.58 & 1643 \\
\hline 0.02 & $8.19 \mathrm{E}-04$ & $3.56 \mathrm{E}-01$ & $1.38 \mathrm{E}-01$ & $3.01 \mathrm{E}-07$ & 1.57 & 1641 \\
\hline 0.05 & $1.83 \mathrm{E}-03$ & $4.24 \mathrm{E}-01$ & $1.65 \mathrm{E}-01$ & $8.03 \mathrm{E}-07$ & 1.56 & 1639 \\
\hline 0.1 & $1.19 \mathrm{E}-03$ & $3.92 \mathrm{E}-01$ & $1.53 \mathrm{E}-01$ & $4.85 \mathrm{E}-07$ & 1.54 & 1659 \\
\hline 0.2 & $1.77 \mathrm{E}-03$ & $2.97 \mathrm{E}-01$ & $1.17 \mathrm{E}-01$ & $5.52 \mathrm{E}-07$ & 1.51 & 1664 \\
\hline 0.4 & $6.06 \mathrm{E}-03$ & $2.13 \mathrm{E}-01$ & $8.47 \mathrm{E}-02$ & $1.37 \mathrm{E}-06$ & 1.47 & 1675 \\
\hline 0.8 & $2.58 \mathrm{E}-03$ & $1.47 \mathrm{E}-01$ & $5.97 \mathrm{E}-02$ & $4.10 \mathrm{E}-07$ & 1.41 & 1697 \\
\hline 1.6 & $2.44 \mathrm{E}-03$ & $9.80 \mathrm{E}-02$ & $4.07 \mathrm{E}-02$ & $2.64 \mathrm{E}-07$ & 1.33 & 1721 \\
\hline 3.2 & $3.25 \mathrm{E}-03$ & $6.32 \mathrm{E}-02$ & $2.71 \mathrm{E}-02$ & $2.35 \mathrm{E}-07$ & 1.23 & 1779 \\
\hline 6.4 & $5.20 \mathrm{E}-03$ & $4.29 \mathrm{E}-02$ & $1.92 \mathrm{E}-02$ & $2.66 \mathrm{E}-07$ & 1.09 & 1875 \\
\hline 12.8 & $4.61 \mathrm{E}-03$ & $1.78 \mathrm{E}-02$ & $8.49 \mathrm{E}-03$ & $1.04 \mathrm{E}-07$ & 0.98 & 1929 \\
\hline 25.6 & $4.07 \mathrm{E}-03$ & $1.04 \mathrm{E}-02$ & $5.28 \mathrm{E}-03$ & $5.70 \mathrm{E}-08$ & 0.84 & 2057 \\
\hline \multicolumn{7}{|c|}{ Section $133-817 \mathrm{~B}-3 \mathrm{H}-4$} \\
\hline 0 & & & & & 1.87 & 1527 \\
\hline 0.01 & & & & & 1.87 & 1526 \\
\hline 0.02 & & $3.24 \mathrm{E}-01$ & $1.13 \mathrm{E}-01$ & & 1.86 & 1530 \\
\hline 0.05 & $5.70 \mathrm{E}-03$ & $3.52 \mathrm{E}-01$ & $1.23 \mathrm{E}-01$ & $1.86 \mathrm{E}-06$ & 1.85 & 1530 \\
\hline 0.1 & $5.64 \mathrm{E}-03$ & $3.07 \mathrm{E}-01$ & $1.08 \mathrm{E}-01$ & $1.62 \mathrm{E}-06$ & 1.84 & 1534 \\
\hline 0.2 & $1.43 \mathrm{E}-02$ & $2.29 \mathrm{E}-01$ & $8.08 \mathrm{E}-02$ & $3.07 \mathrm{E}-06$ & 1.81 & 1545 \\
\hline 0.4 & $9.71 \mathrm{E}-03$ & $1.60 \mathrm{E}-01$ & $5.70 \mathrm{E}-02$ & $1.47 \mathrm{E}-06$ & 1.78 & 1545 \\
\hline 0.8 & $1.36 \mathrm{E}-02$ & $1.08 \mathrm{E}-01$ & $3.88 \mathrm{E}-02$ & $1.41 \mathrm{E}-06$ & 1.74 & 1570 \\
\hline 1.6 & $2.05 \mathrm{E}-02$ & $7.42 \mathrm{E}-02$ & $2.71 \mathrm{E}-02$ & $1.48 \mathrm{E}-06$ & 1.68 & 1594 \\
\hline 3.2 & $1.95 \mathrm{E}-02$ & $4.80 \mathrm{E}-02$ & $1.79 \mathrm{E}-02$ & $9.28 \mathrm{E}-07$ & 1.60 & 1616 \\
\hline 6.4 & $1.81 \mathrm{E}-02$ & $3.46 \mathrm{E}-02$ & $1.33 \mathrm{E}-02$ & $6.40 \mathrm{E}-07$ & 1.49 & 1656 \\
\hline 12.8 & $1.64 \mathrm{E}-02$ & $2.02 \mathrm{E}-02$ & $8.09 \mathrm{E}-03$ & $3.54 \mathrm{E}-07$ & 1.36 & 1723 \\
\hline 25.6 & $3.68 \mathrm{E}-02$ & $1.47 \mathrm{E}-02$ & $6.20 \mathrm{E}-03$ & 6.07E-07 & 1.18 & 1820 \\
\hline
\end{tabular}

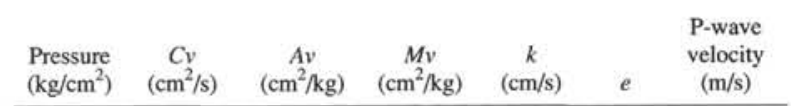

Section 133-817B-6H-4

$\begin{array}{lll}0 & & \\ 0.01 & & \\ 0.02 & 1.10 \mathrm{E}-03 & 1.25 \mathrm{E}-01 \\ 0.05 & 9.85 \mathrm{E}-03 & 1.79 \mathrm{E}-01 \\ 0.1 & 7.19 \mathrm{E}-03 & 1.80 \mathrm{E}-01 \\ 0.2 & 1.40 \mathrm{E}-02 & 1.36 \mathrm{E}-01 \\ 0.4 & 1.38 \mathrm{E}-02 & 9.86 \mathrm{E}-02 \\ 0.8 & 2.11 \mathrm{E}-02 & 6.74 \mathrm{E}-02 \\ 1.6 & 2.06 \mathrm{E}-02 & 4.65 \mathrm{E}-02 \\ 3.2 & 1.99 \mathrm{E}-02 & 3.05 \mathrm{E}-02 \\ 6.4 & 3.35 \mathrm{E}-02 & 2.26 \mathrm{E}-02 \\ 12.8 & 3.08 \mathrm{E}-02 & 1.89 \mathrm{E}-02 \\ 25.6 & 2.77 \mathrm{E}-02 & 8.02 \mathrm{E}-03\end{array}$

$\begin{array}{lll} & & 1.51 \\ 4.98 \mathrm{E}-02 & 1.45 \mathrm{E}-07 & 1.51 \\ 7.15 \mathrm{E}-02 & 1.87 \mathrm{E}-06 & 1.50 \\ 7.20 \mathrm{E}-02 & 1.38 \mathrm{E}-06 & 1.49 \\ 5.47 \mathrm{E}-02 & 2.03 \mathrm{E}-06 & 1.48 \\ 3.98 \mathrm{E}-02 & 1.46 \mathrm{E}-06 & 1.46 \\ 2.74 \mathrm{E}-02 & 1.54 \mathrm{E}-06 & 1.43 \\ 1.91 \mathrm{E}-02 & 1.05 \mathrm{E}-06 & 1.39 \\ 1.28 \mathrm{E}-02 & 6.74 \mathrm{E}-07 & 1.34 \\ 9.62 \mathrm{E}-03 & 8.59 \mathrm{E}-07 & 1.27 \\ 8.33 \mathrm{E}-03 & 6.82 \mathrm{E}-07 & 1.15 \\ 3.73 \mathrm{E}-03 & 2.75 \mathrm{E}-07 & 1.05\end{array}$

1594

1592

1592
1595

1602

1606

1628

1659

1659
1716
1716

1800

1853

Section 133-817B-10H-4

0

0

$\begin{array}{llllll}0.01 & & & & & 1.24 \\ 0.02 & & 1.97 \mathrm{E}-01 & 8.76 \mathrm{E}-02 & & 1.24 \\ 0.05 & 5.18 \mathrm{E}-03 & 2.00 \mathrm{E}-01 & 8.94 \mathrm{E}-02 & 1.25 \mathrm{E}-06 & 1.24\end{array}$

$\begin{array}{llllll}0.1 & 6.72 \mathrm{E}-03 & 1.64 \mathrm{E}-01 & 7.34 \mathrm{E}-02 & 1.33 \mathrm{E}-06 & 1.23\end{array}$

$\begin{array}{llllll}0.2 & 1.31 \mathrm{E}-02 & 1.09 \mathrm{E}-01 & 4.88 \mathrm{E}-02 & 1.72 \mathrm{E}-06 & 1.22\end{array}$

$\begin{array}{lllllll}0.2 & 1.32 \mathrm{E}-02 & 1.09 \mathrm{E}-02 & 3.57 \mathrm{E}-02 & 1.24 \mathrm{E}-06 & 1.20\end{array}$

$\begin{array}{llllll}0.4 & 1.29 \mathrm{E}-02 & 7.92 \mathrm{E}-02 & 3.57 \mathrm{E}-02 & 1.24 \mathrm{E}-06 & 1.20 \\ 0.8 & 1.57 \mathrm{E}-02 & 5.49 \mathrm{E}-02 & 2.49 \mathrm{E}-02 & 1.05 \mathrm{E}-06 & 1.18\end{array}$

$\begin{array}{lllllll}0.8 & 1.57 \mathrm{E}-02 & 5.49 \mathrm{E}-02 & 2.49 \mathrm{E}-02 & 1.05 \mathrm{E}-06 & 1.18 \\ 1.6 & 1.93 \mathrm{E}-02 & 3.90 \mathrm{E}-02 & 1.79 \mathrm{E}-02 & 9.34 \mathrm{E}-07 & 1.15\end{array}$

$\begin{array}{llllll}3.2 & 1.87 \mathrm{E}-02 & 2.71 \mathrm{E}-02 & 1.26 \mathrm{E}-02 & 6.36 \mathrm{E}-07 & 1.11\end{array}$

$\begin{array}{llllll}6.4 & 1.78 \mathrm{E}-02 & 1.79 \mathrm{E}-02 & 8.50 \mathrm{E}-03 & 4.08 \mathrm{E}-07 & 1.05\end{array}$

$\begin{array}{llllll}12.8 & 2.19 \mathrm{E}-02 & 1.10 \mathrm{E}-02 & 5.37 \mathrm{E}-03 & 3.16 \mathrm{E}-07 & 0.98\end{array}$

$\begin{array}{llllll}12.8 & 2.72 \mathrm{E}-02 & 8.36 \mathrm{E}-03 & 4.23 \mathrm{E}-03 & 3.10 \mathrm{E}-07 & 0.87\end{array}$

1542

1547

1552
1548

1548

1547

1555

1578
1602

1602
1604

1655

1798

Section 133-817B-13H-4

0
0.01

0.01
0.02

0.02
0.05

$\begin{array}{lll}0.05 & 6.77 \mathrm{E}-03 & 1.11 \mathrm{E}-01 \\ 0.1 & 9.18 \mathrm{E}-03 & 1.02 \mathrm{E}-01\end{array}$

$0.2 \quad 1.31 \mathrm{E}-02 \quad 7.06 \mathrm{E}-02$

$\begin{array}{lll}0.2 & 1.31 \mathrm{E}-02 & 7.06 \mathrm{E}-02 \\ 0.4 & 6.08 \mathrm{E}-02\end{array}$

$\begin{array}{lll}0.4 & 2.03 \mathrm{E}-02 & 6.08 \mathrm{E}-02 \\ 0.8 & 2.00 \mathrm{E}-02 & 4.21 \mathrm{E}-02\end{array}$

$1.6 \quad 2.56 \mathrm{E}-02 \quad 3.08 \mathrm{E}-02$

3.2 2.49E-02 $1.90 \mathrm{E}-02$

$\begin{array}{lll}6.4 & 2.40 \mathrm{E}-02 & 1.28 \mathrm{E}-02 \\ 12.8 & 3.11 \mathrm{E}-02 & 8.41 \mathrm{E}-03\end{array}$

$\begin{array}{lll}12.8 & 3.11 \mathrm{E}-02 & 8.41 \mathrm{E}-03 \\ 25.6 & 2.87 \mathrm{E}-02 & 7.25 \mathrm{E}-03\end{array}$

$\begin{array}{lll}4.23 \mathrm{E}-03 & 3.10 \mathrm{E}-07 \quad 0.87\end{array}$

Section 133-817B-16H-4

$\begin{array}{lll}0 & & \\ 0.01 & & \\ 0.02 & & 1.74 \mathrm{E}-01 \\ 0.05 & 6.45 \mathrm{E}-03 & 1.16 \mathrm{E}-01 \\ 0.1 & 8.74 \mathrm{E}-03 & 1.17 \mathrm{E}-01 \\ 0.2 & 8.69 \mathrm{E}-03 & 5.77 \mathrm{E}-02 \\ 0.4 & 8.62 \mathrm{E}-03 & 5.22 \mathrm{E}-02 \\ 0.8 & 1.23 \mathrm{E}-02 & 3.07 \mathrm{E}-02 \\ 1.6 & 1.49 \mathrm{E}-02 & 2.69 \mathrm{E}-02 \\ 3.2 & 2.40 \mathrm{E}-02 & 2.01 \mathrm{E}-02 \\ 6.4 & 2.30 \mathrm{E}-02 & 1.49 \mathrm{E}-02 \\ 12.8 & 2.18 \mathrm{E}-02 & 8.89 \mathrm{E}-03 \\ 25.6 & 2.35 \mathrm{E}-02 & 5.98 \mathrm{E}-03\end{array}$

$\begin{array}{llll} & & 1.02 & 1593 \\ & & 1.02 & 1592 \\ 9.27 \mathrm{E}-02 & & 1.02 & 1597 \\ 5.50 \mathrm{E}-02 & 1.01 \mathrm{E}-06 & 1.01 & 1597 \\ 5.06 \mathrm{E}-02 & 1.25 \mathrm{E}-06 & 1.01 & 1597 \\ 3.52 \mathrm{E}-02 & 1.25 \mathrm{E}-06 & 1.00 & 1598 \\ 3.04 \mathrm{E}-02 & 1.67 \mathrm{E}-06 & 0.99 & 1608 \\ 2.12 \mathrm{E}-02 & 1.15 \mathrm{E}-06 & 0.97 & 1622 \\ 1.56 \mathrm{E}-02 & 1.08 \mathrm{E}-06 & 0.95 & 1636 \\ 9.77 \mathrm{E}-03 & 6.57 \mathrm{E}-07 & 0.92 & 1662 \\ 6.66 \mathrm{E}-03 & 4.31 \mathrm{E}-07 & 0.88 & 1711 \\ 4.48 \mathrm{E}-03 & 3.76 \mathrm{E}-07 & 0.82 & 1762 \\ 3.98 \mathrm{E}-03 & 3.07 \mathrm{E}-07 & 0.73 & 1828\end{array}$

Section 133-817B-20H-4

$\begin{array}{lllllll}0 & & & & & 1.15 \\ 0.01 & & & & & 1.15 \\ 0.02 & & 2.69 \mathrm{E}-01 & 1.25 \mathrm{E}-01 & & 1.15 \\ 0.05 & 7.15 \mathrm{E}-03 & 2.80 \mathrm{E}-01 & 1.30 \mathrm{E}-01 & 2.51 \mathrm{E}-06 & 1.14 \\ 0.1 & 9.64 \mathrm{E}-03 & 2.32 \mathrm{E}-01 & 1.09 \mathrm{E}-01 & 2.83 \mathrm{E}-06 & 1.13 \\ 0.2 & 9.51 \mathrm{E}-03 & 1.67 \mathrm{E}-01 & 7.84 \mathrm{E}-02 & 2.01 \mathrm{E}-06 & 1.11 \\ 0.4 & 9.33 \mathrm{E}-03 & 1.19 \mathrm{E}-01 & 5.63 \mathrm{E}-02 & 1.42 \mathrm{E}-06 & 1.09 \\ 0.8 & 1.31 \mathrm{E}-02 & 6.86 \mathrm{E}-02 & 3.29 \mathrm{E}-02 & 1.16 \mathrm{E}-06 & 1.06 \\ 1.6 & 1.27 \mathrm{E}-02 & 4.85 \mathrm{E}-02 & 2.36 \mathrm{E}-02 & 8.08 \mathrm{E}-07 & 1.02 \\ 3.2 & 1.50 \mathrm{E}-02 & 3.18 \mathrm{E}-02 & 1.57 \mathrm{E}-02 & 6.37 \mathrm{E}-07 & 0.97 \\ 6.4 & 1.79 \mathrm{E}-02 & 1.91 \mathrm{E}-02 & 9.72 \mathrm{E}-03 & 4.70 \mathrm{E}-07 & 0.91 \\ 12.8 & 1.68 \mathrm{E}-02 & 9.80 \mathrm{E}-03 & 5.14 \mathrm{E}-03 & 2.32 \mathrm{E}-07 & 0.84 \\ 25.6 & 1.55 \mathrm{E}-02 & 6.39 \mathrm{E}-03 & 3.46 \mathrm{E}-03 & 1.45 \mathrm{E}-07 & 0.76\end{array}$

1638

1644

1647

1647
1647

1647
1647

1647
1647
1656

1676

1678

1738
1795

1883

Note: $C y=$ coefficient of consolidation; $A v=$ coefficient of compressibility; $M v=$ coefficient of volume change; $k=$ coefficient of permeability; $e=$ void ratio. 


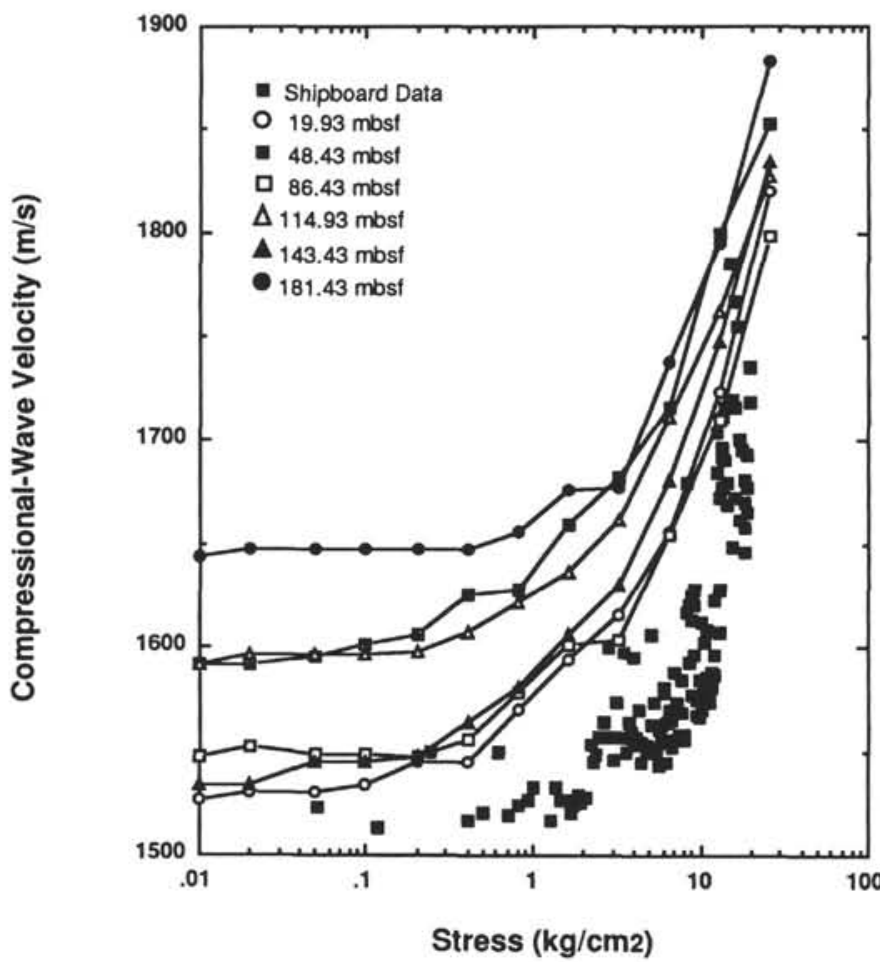

Figure 9. Compressional-wave velocity vs. effective stress for Site 817 consolidation samples and comparison of shipboard measurements.

sional-wave velocity measurements. The latter measurement was obtained during the consolidation test. The regression equations for both Sites 815 and 817 between bulk modulus ratio and effective stress are $Y=1.0075+0.09 X, Y=1.059+0.02087 X$, respectively. The increasing rate in the Site 815 sample is almost 4.5 times that in the Site 817 sample. This is why compressional-wave velocity is offset between these two sites during an overloading stress below 10 $\mathrm{kg} / \mathrm{cm}^{2}$ (Fig. 5). At about $15 \mathrm{~kg} / \mathrm{cm}^{2}$, effective stress (about $195 \mathrm{mbsf}$ in Site 815; about 210 mbsf in Site 817), the shipboard-measured compressional-wave velocities of both sites are almost the same because the chalk appears common in this depth for both sites. The clay content in the sediment will influence the compressional-wave velocity and elastic properties considerably.

\section{CONCLUSIONS}

At Site 815 , the top $10 \mathrm{~m}$ of sediments shows that porosities increase along the depth in the core because the hydrographic pressure within the pore water had been released when the sediments were sampled from the seafloor and brought on board the ship. At Site 817, this collapsing effect did not occur in the top sediments of core.

The nonlithified sediments at Site 815 are composed mainly of clay mixing with pelagic nannofossil or foraminifers oozes. These derived from Australian mainland clay and were deposited only on the northwestern edge of Marion Plateau during the early Pliocene. Perhaps, this clay did not cross the Townsville Trough.

The physical properties in the carbonate sediments at both sites can be highly correlated with burial depth or overloading pressure. The clay-rich sediments at Site 815 maintain a much lower rate of almost constant value along depth in the core than those at Site 817. The reason is not only because of the faster depositional rate at Site 815 during early Pliocene, but the clay may have maintained more abundant interstitial water in the sediments. Nevertheless, the compressional-wave velocity maintains more or less constant increasing rates at both sites. Above $100 \mathrm{mbsf}$, the velocity rate in the Site 815 sample is almost $100 \mathrm{~m} / \mathrm{s}$ faster than that of sediment from Site 817 .

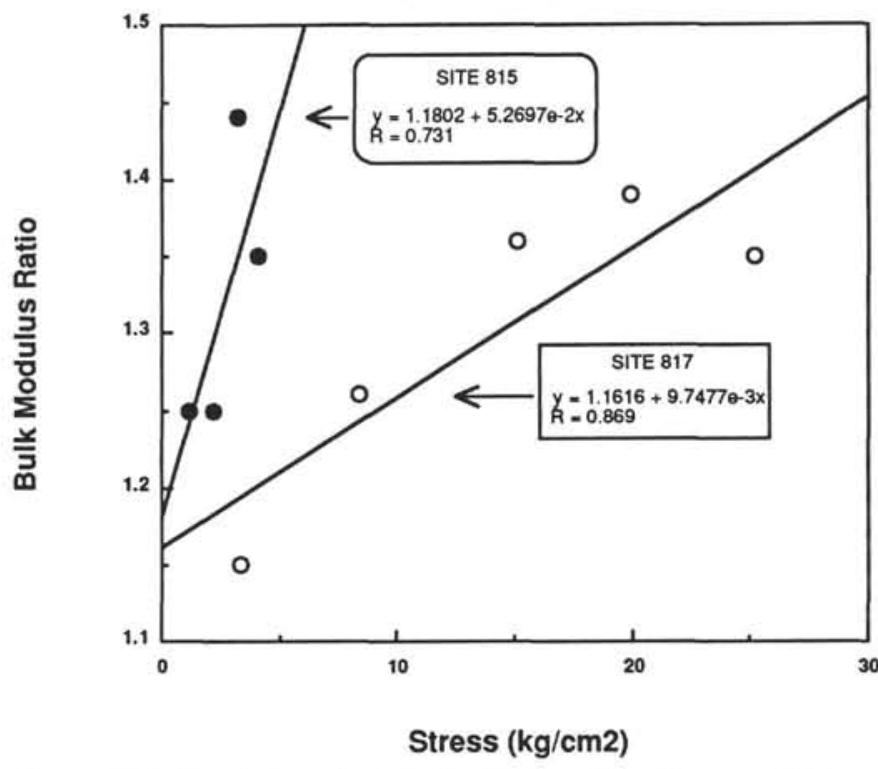

Figure 10. Bulk modulus ratio (calculated bulk modulus from consolidation test with the results of shipboard measurements) vs. effective stress for Sites 815 and 817 consolidation samples.

Below a depth of about $200 \mathrm{mbsf}$, these carbonate sediments at both sites start to become chalk, and their acoustic and elastic properties no longer are influenced by the clay content.

During the consolidation test, we show that sediments having dominant micritic particles may have faster acoustic velocity than sediment that is composed mainly of coccoliths.

The bulk modulus ratio increasing rate in the clay-rich carbonate sediments is almost 4.5 times higher than in the clay-free periplatform carbonate sediments. Clay particles are an important part of the compaction effects in these carbonate sediments.

\section{ACKNOWLEDGMENTS}

We thank the fine staff of the Ocean Drilling Program and our colleague, Amanda Palmer-Julson, for her help in the shipboard laboratory. We are grateful to Philip D. Rabinowitz, Director of ODP, for allowing Min-Pen Chen to participate during Leg 133, otherwise, this study would not have been completed. This study was funded by the National Science Council of the Republic of China, under Grant NSC80-0209-M002a-03.

\section{REFERENCES $*$}

Boyce, R.E., 1976. Definitions and laboratory techniques of compressional sound velocity parameters and wet-water content, wet-bulk density, and porosity parameters by gravimetric and gamma ray attenuation techniques. In Schlanger, S.O., Jackson, E.D., et al., Init. Repts. DSDP, 33: Washington (U.S. Govt. Printing Office), 931-958.

Bryant, W.R., Cernock, P., and Morelock, J., 1967. Shear strength and consolidation characteristics of marine sediments from the western Gulf of Mexico. In Richards, A.F. (Ed.), Marine Geotechnique: Chicago (Univ. of Illinois Press), 41-62.

Bryant, W.R., Deflanche, A.P., and Trabant, P.K., 1974. Consolidation and marine clays and carbonates. In Inderbitzen, A.L. (Ed.), Deep-Sea Sediments: New York (Plenum), 209-144.

Davies, P.J., 1989. The evolution of the carbonate platforms of northeast Australia. Spec. Publ.-Soc. Econ. Paleontol. Mineral., 44:233-258.

Davies, P.J., McKenzie, J.A., Palmer-Julson, A., et al., 1991. Proc. ODP, Init. Repts., 133: College Station, TX (Ocean Drilling Program).

Abbreviations for names of organizations and publication titles in ODP reference lists follow the style given in Chemical Abstracts Service Source Index (published by American Chemical Society). 
Demars, K.R., 1982. Unique engineering properties and compression behavior of deep-sea calcareous sediments. In Demars, K.R., and Chaney, R.C. (Eds.), Geotechnical Properties, Behavior, and Performance of Calcareous Soils. ASTM Spec. Tech. Publ., 777:97-112.

Folk. R.L., 1974. Petrology of Sedimentary Rocks: Austin (Hemphill Publ.).

Hamilton, E., 1979. $V_{\mathrm{p}} / V_{\mathrm{s}}$ and Poisson's ratios in marine sediments and rocks. J. Acoust. Soc. Am., 66:1093-1101.

Lavoie, D., 1988. Geotechnical properties of sediments in a carbonate-slope environment: Ocean Drilling Program Site 630, Northern Little Bahama Bank. In Austin, J.A., Jr., Schlager, W., et al., Proc. ODP, Sci. Results, 101: College Station, TX (Ocean Drilling Program), 315-326.

Lee, H.J., 1982. Bulk density and shear strength of several deep-sea calcareous sediments. In Demars, K.R., and Chaney, R.C. (Eds.), Geotechnical Prop- erties, Behavior, and Performance of Calcareous Soils. ASTM Spec. Tech. Publ., 777:54-78.

McClelland, B., 1967. Progress of consolidation in delta front and prodelta clays of the Mississippi River. In Richards, A.F. (Ed.), Marine Geotechnique: Chicago (Univ. of Illinois Press), 22-40.

Molnia, B.F., 1974. A rapid and accurate method for the analysis of calcium carbonate in small samples. J. Sediment. Petrol., 44:589-590.

Date of initial receipt: 9 April 1992

Date of acceptance: 14 January 1993

Ms 133SR-266 